\section{Capture every detail in two minutes}

\begin{abstract}
New 3M Impregum Super Quick Polyether Impression Material the first true superfast polyether - combines the trusted performance and reliability of a polyether with the speed of a VPS. It is available in two consistencies, medium and light body, and can be used in the monophase and 1-step impression techniques. Both materials have a
\end{abstract} working time of 45 seconds

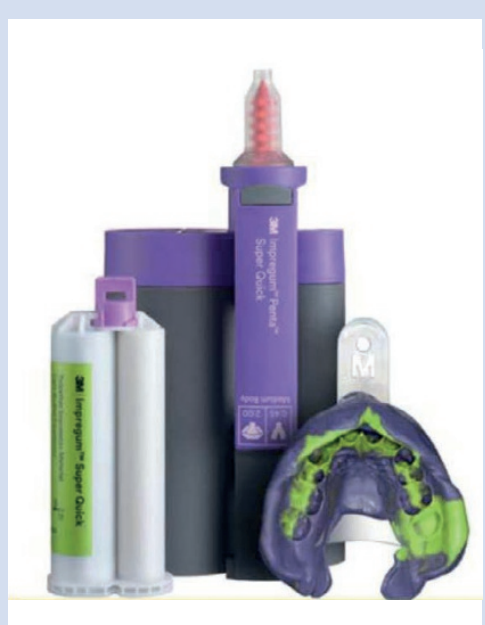
and an intra-oral setting time of two minutes, making them perfectly suited for single-unit restorations like crowns, implants, inlays/onlays and smaller bridges up to three units.

The short intra-oral setting time, combined with a significantly improved fresh, minty taste, provide increased patient comfort. The new material also has the trusted polyether benefits: moisture tolerance, great flowability and a consistent flow throughout the whole working time. They ensure highly detailed and accurate impressions.

The material is particularly suited for use in smaller cases. The hallmark polyether properties such as intrinsic hydrophilicity, unique flowability and specific setting behaviour remain unchanged.

For more information visit https://www.3m. co.uk/3M/en_GB/dental-uk/products/impressioning/ impregumsuper-fast-polyether-impression/.

\section{Your reputation precedes you}

\section{The Enlighten Whitening system is} the only tooth whitening solution to guarantee a Vita shade B1 for every patient. With the aim of improving the standard of tooth whitening treatment in the UK, the team at Enlighten want to go one step further than offering a superior product - they want to provide the chance for aspiring practitioners to become the leading tooth whitening authority in their local area.

By joining Enlighten's Regional Centres of Excellence Scheme, practices can benefit from exclusive training to ensure the entire dental team have the knowledge and skills to become experts in tooth whitening - able to answer patients' queries with ease.

With their own network of contacts, Enlighten will help to market your expertise effectively, to ensure that your practice develops an astounding reputation for quality service with maximum results.

For more information, visit www.enlightensmiles.com, email info@ enlightensmiles.com or call 02074243270.

\section{Become an 'autism-friendly' practice}

One in ten people in the UK is diagnosed with autism and the figure is set to rise. For these people smells, bright lights, invasion of personal space and an unfamiliar environment are some of the many triggers that can induce high levels of anxiety and stress. In turn this can lead to panic and unpredictable behaviour, and potential emotional meltdowns.

It can be difficult for members of staff to know how best to handle such a situation, which can not only be upsetting for the individual but also for friends, family and clients visiting the practice, leading to a poor patient experience.

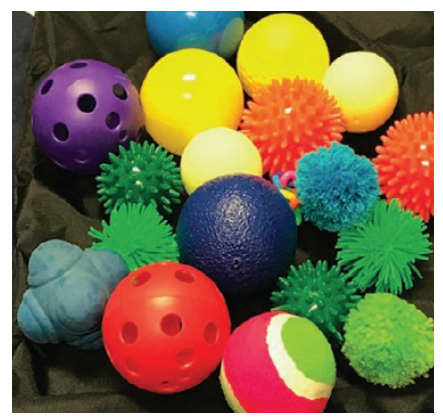

To help reduce anxiety and stress, Pulse supplies dental and GP practices and police forces across the UK with 'Autism Care Boxes', which help in reducing anxiety and stress.

'Autism Care Boxes' contain various aids from a weighted blanket and knee blanket to specialist tangles and sensory aids to help reduce anxiety and stress for individuals. They are easily kept behind a reception desk and every item in the box can be wiped down for reasons of hygiene and cleanliness.

By providing an 'Autism Care Box' in your practice, you can help individuals cope with their anxiety and behaviour, as well as giving staff the tools to deal with a potentially difficult situation. You are also championing the fact that you are an 'Autism Friendly' practice which may set you apart from other dental practices in the area.

For further information contact 07976727573 or 01614286042.

\section{Hassle-free handpiece repairs}

Dental Express has become one of the UK's leading suppliers of high-quality dental consumables. The expert team strives to deliver customer service that is second-to-none, but did you know they also offer a dedicated handpiece repair service?

Dental Express keeps affordability in mind by offering free evaluations with a $100 \%$ no-risk policy. If you decide to proceed with repairs, Dental Express guarantees all work carried out, so you can rest assured that your handpiece will be returned to you in supreme-working condition.

You can also feel confident knowing Dental Express provides this exceptional service with a same-day turnaround, enabling you to avoid delays that could otherwise stall the provision of high quality dental care.

Keep your surgery running smoothly with an efficient and cost-effective handpiece repair service from Dental Express.

For more information, visit www.dental-express.co.uk, call on 08007076212 or contact martin.oates@dental-express.co.uk. 